\title{
ON DISTRIBUTED PARAMETER CONTROL SYSTEMS IN THE ABNORMAL CASE AND IN THE CASE OF NONOPERATOR EQUALITY CONSTRAINTS ${ }^{1}$
}

\author{
URSZULA LEDZEWICZ ${ }^{2}$ \\ Southern Illinois University at Edwardsville \\ Department of Mathematics and Statistics \\ Edwardsville, IL 62026, U.S.A.
}

\begin{abstract}
In this paper, a general distributed parameter control problem in Banach spaces with integral cost functional and with given initial and terminal data is considered. An extension of the Dubovitskii-Milyutin method to the case of nonregular operator equality constraints, based on Avakov's generalization of the Lusternik theorem, is presented. This result is applied to obtain an extension of the Extremum Principle for the case of abnormal optimal control problems. Then a version of this problem with nonoperator equality constraints is discussed and the Extremum Principle for this problem is presented.
\end{abstract}

Key words: Distributed parameter control system, Lusternik theorem, abnormal process, Dubovitskii-Milyutin method, nonoperator equality constraints, Extremum Principle.

AMS (MOS) subject classifications: $\quad 49 \mathrm{~K} 27,49 \mathrm{~K} 10$.

\section{INTRODUCTION}

Results on optimal control of distributed parameter problems have been considered in several papers starting with Lions [7]. Lions' results have been extended to the case of cost functionals in the integral form [5, 6]. In [8], Lions considered a distributed parameter control problem with the initial condition not a priori given; (so called "system with insufficient data”). In [14], Papageorgiou presented a significant generalization of Lions' results to the case of a general convex integral functional. In a general setting of Banach spaces, by using the Dubovitskii-Milyutin method, he proved necessary and sufficient conditions of optimality which generalized several results previously given $[5,6]$.

\footnotetext{
${ }^{1}$ Received: January, 1993. Revised: May, 1993.

${ }^{2}$ Research supported by NSF grant DMS-9109324, by SIUE Research Scholar Award and Fourth Quarter Fellowship, Summer 1992.
} 
In this paper, a general distributed parameter control problem in a Banach space with integral cost functional, as formulated by Papageorgiou in [14], is considered but in the case of given initial and terminal data (Section 2). For this problem, the case of abnormal optimal processes, i.e. processes for which the Extremum Principle is satisfied in the trivial way with multiplier $\lambda_{0}$ corresponding to the cost functional equal to zero, is discussed. An extension of the Dubovitskii-Milyutin method to the case of nonregular operator equality constraints from [11] obtained by the application of Avakov's results [1] is briefly presented in Section 3. This extension has already been applied to an abnormal optimal control problem of a system governed by ordinary differential equations with given terminal data in [12]. Extended versions of the local Maximum Principle for this problem have been obtained in [2] and [12]. In this paper, following the idea of [12], the result of [11] is applied to the problem from Section 2 and an extension of the Extremum Principle for the distributed parameter control system with given initial and terminal data in the abnormal case is formulated (Section 4).

In the last part of the paper (Section 5), the optimal control problem from Section 2 is considered but without terminal data and without the assumption that the control set possesses a nonempty interior in $L^{2}(Y)$, i.e., this is a problem with a nonoperator equality constraint. By using a generalization of the Dubovitski-Milyutin method to the case of $n$ equality constraints in arbitrary, even nonoperator form from [15], a version of the Extremum Principle is proved generalizing results of [5] and [10].

\section{A GENERAL OPTIMAL CONTROL PROBLEM IN A BANACH SPACE}

Consider the following general optimal control problem as considered by Papageorgiou [14], but with fixed initial and terminal data. Minimize the functional

under the constraints

$$
I(x, u)=\int_{0}^{b} f(t, x(t), u(t)) d t
$$

$$
\begin{aligned}
\dot{x}(t)+A(t, x(t)) & =B(t, x(t)) u(t) \\
x(0) & =x_{0} \\
x(b) & =x_{1} \\
x(t) & \in U(t)
\end{aligned}
$$

where $x \in X \subset H ; H$ is a separable Hilbert space, $X$ is a separable reflexive Banach space embedded continuously and densely in $H ; u \in Y$ and $Y$ is a separable Banach space modeling 
the control space; $x_{0}, x_{1} \in H$.

In order to specify the assumptions of the problem, we need to introduce the following notations. Let $X^{*}$ denote the dual space to $X$ and let $\|\cdot\|,|\cdot|,\|\cdot\|_{*}$ be norms in the spaces $X, H, X^{*}$ respectively. By $(\cdot, \cdot)$ we will denote the inner product in $H$ and by $\langle\cdot, \cdot\rangle$ the value of the functionals from $X^{*}$ on $X$ or from $Y^{*}$ on $Y$. Then for every $x \in X \subset H$ and $h \in H \subset X^{*}$ we have $(x, h)=\langle h, x\rangle$.

Suppose $A:[0, b] \times X \rightarrow X^{*}, B:[0, b] \times H \rightarrow L\left(Y, X^{*}\right)$ are operators, $f:[0, b] \times H \times Y \rightarrow R$ is a functional, $U:[0, b] \rightarrow P(Y)$ is a multifunction with $P(Y)$ a nonempty, closed, convex subset of $Y$.

The problem above will be considered under the following assumptions:

(A1) $t \rightarrow A(t, x)$ is measurable;

(A2) $x \rightarrow A(t, x)$ is twice continuously Fréchet differentiable and strongly monotone uniformly in $t \in T$;

(A3) $\|A(t, x)\|_{*} \leq a(t)+b\|x\|$ a.e. with $a(\cdot) \in L_{+}^{2}, b>0$

(A4) $\langle A(t, x), x\rangle \geq c\|x\|^{2}$, with $c<0$;

(A5) $t \rightarrow B(t, x) u$ is measurable for every $(x, u) \in H \times Y$;

(A6) $x \rightarrow B(t, x)$ is continuous;

(A7) $\quad x \rightarrow B(t, x) u$ is twice continuously Fréchet differentiable;

$$
\|B(t, x) u\|_{*} \leq b_{1}(t)+b_{2}(t)|x|+b_{3}(t)\|u\| \quad \text { with } \quad b_{1}(\cdot), \quad b_{2}(\cdot) \in L_{+}^{2},
$$
$b_{3}(\cdot) \in L_{+}^{\infty}$

(A9) $\quad t \rightarrow f(t, x, u)$ is measurable;

(A10) $(x, u) \rightarrow f(t, x, u)$ is continuously Fréchet differentiable;

(A11) int $V \neq \emptyset$ where $V=\left\{u(\cdot) \in L^{2}(Y): u(t) \in U(t)\right.$ a.e. $\}$;

(A12) $U:[0, b] \rightarrow P(Y)$ is a multifunction such that the set $\{(t, u) \in[0, b] \times Y: u \in U(t)\} \in$ $B([0, b]) \times B(Y)$, where $B([0, b])$ is the Borel $\sigma$-field of $[0, b], B(Y)$ is a Borel $\sigma$ field of $Y$.

Let us call the problem (2.1)-(2.5), under assumptions $(A 1)-(A 12)$, Problem I. These assumptions are basically the same as in [14] except for the stronger requirement of twice Fréchet differentiability in $(A 2)$ and $(A 7)$ related to applications of results from Section 3.

We will look for a solution of the above problem in the space

$$
W[0, b]=\left\{x(\cdot) \in L^{2}(X): \dot{x}(\cdot) \in L^{2}\left(X^{*}\right)\right\} .
$$

$W[0, b]$ is a Banach space with norm 


$$
\|x\|_{W[0, b]}=\left(\int_{0}^{b}\|x(t)\|^{2} d t+\int_{0}^{b}\|\dot{x}(t)\|_{*}^{2} d t\right)^{\frac{1}{2}} .
$$

\section{AN EXTENSION OF THE DUBOVITSKII-MILYUTIN METHOD TO THE NONREGULAR CASE}

In [1], Avakov generalized the Lusternik theorem to the case of nonregular operators under twice Fréchet differentiability assumption. In [11], this generalization was applied to extend the Dubovitskii-Milyutin method to the case of $m$ equality constraints given by nonregular operators. Now we will present the main result of [11] which will be applied in our investigation of Problem $I$.

Let us consider the following optimization problem: Minimize the functional

$$
I(x) \rightarrow \min
$$

where $I: X \rightarrow R, X$ a Banach space, under the constraints $x \in Z_{i}, i=1,2, \ldots, n, n+1, \ldots, n+m$. Here $Z_{i}, i=1,2, \ldots, n$, represent inequality constraints $\left(i n t Z_{i} \neq \emptyset\right)$, which for $i=1,2, \ldots, k$ are of the form $Z_{i}=\left\{x \in X: \phi_{i}(x) \leq 0\right\}$, where $\phi_{i}, i=1,2, \ldots, k$ are differentiable functionals on $X$, for $i=k+1, \ldots, n$ are convex sets, while $Z_{n+j}=\left\{x \in X: F_{j}(x)=0\right\}, j=1,2, \ldots, m$, where $F_{j}: X \rightarrow Y_{j}$ are given operators and $Y_{1}, Y_{2}, \ldots, Y_{m}$ are Banach spaces.

Define an operator $F: X \rightarrow Y$, where $Y=Y_{1} \times Y_{2} \times \ldots \times Y_{m}$, by taking

$$
F(x)=\left(F_{1}(x), F_{2}(x), \ldots, F_{m}(x)\right)
$$

Let $x_{0} \in X$. We assume that $F$ given by $(3.1)$ is twice Fréchet differentiable at the point $x_{0}$.

For arbitrary $h \in X$ we introduce a linear mapping $G\left(x_{0}, h\right): X \rightarrow Y \times Y / \operatorname{Im} F^{\prime}\left(x_{0}\right)$ given by the formula

$$
G\left(x_{0}, h\right)=\left(F^{\prime}\left(x_{0}\right), \pi F^{\prime \prime}\left(x_{0}\right)(h)\right)
$$

where $\pi: Y \rightarrow Y / \operatorname{Im} F^{\prime}\left(x_{0}\right)$ is the quotient map from $Y$ in to the quotient space $Y / \operatorname{Im} F^{\prime}\left(x_{0}\right)$. We then denote

$$
\begin{aligned}
& W=Y \times Y / \operatorname{Im} F^{\prime}\left(x_{0}\right) \\
& W_{1}=I m F^{\prime}\left(x_{0}\right) \times Y / I m F^{\prime}\left(x_{0}\right) \text {. }
\end{aligned}
$$

In [11], the above optimization problem was considered and by using Avakov's generalization of the Lusternik theorem to the case of nonregular operators from [1], the 
extended version of the Dubovitskii-Milyutin theorem to the case of $m$ equality constraints in the nonregular case was proved in the following form.

Theorem 3.1 [11]: Suppose that

(i) $\quad I(x)$ attains its local minimum on the set $\bigcap_{i=1}^{n+m} Z_{i}$ at the point $x_{0}$;

(ii) $\quad I(x)$ is Fréchet differentiable at $x_{0}$ with $I^{\prime}\left(x_{0}\right) \neq 0$ and cone of decrease $C_{0}$;

(iii) $\phi_{i}, i=1,2, \ldots, k$ are Fréchet differentiable at $x_{0}$ with $\phi_{i}^{\prime}\left(x_{0}\right) \neq 0$ if $\phi_{i}\left(x_{0}\right)=0$ and the inequality constraints $Z_{i}$ have feasible cones $C_{i}, i=1,2, \ldots, n$ at the point $x_{0}$;

(iv) the operator $F$ given in (3.1) is twice Fréchet differentiable at $x_{0}$ and $\operatorname{Im} F^{\prime}\left(x_{0}\right)$ is closed.

Then for every $h \in \bigcap_{i=0}^{k} \operatorname{clos} C_{i}$ such that

$h \in \operatorname{ker} F_{j}^{\prime}\left(x_{0}\right), j=1,2, \ldots, m, \pi\left(F_{1}^{\prime \prime}\left(x_{0}\right)(h) h, \ldots, F_{m}^{\prime \prime}\left(x_{0}\right)(h) h\right)=0$

for which the second order feasible cones at $x_{0}$ to $Z_{i}, i=k+1, \ldots, n$, in the direction of $h$ are nonempty and convex, and for which $\operatorname{Im} G\left(x_{0}, h\right)$ is closed in $W$, there exist $2 m+n+1$ functionals, not all zero,

$$
f_{i}(h) \in C_{i}^{*}, i=0,1, \ldots, n, q_{j}^{*}(h), s_{j}^{*}(h) \in Y_{j}^{*}, j=1,2, \ldots, m,
$$

such that

and

$$
\sum_{i=0}^{n} f_{i}(h)+\sum_{j=1}^{m} F_{j}^{\prime *}\left(x_{0}\right) q_{j}^{*}(h)+\sum_{j=1}^{m}\left(F_{j}^{\prime \prime}\left(x_{0}\right)(h)\right)^{*} s_{j}^{*}(h)=0
$$

$$
\left(s_{1}^{*}(h), \ldots, s_{m}^{*}(h)\right) \in\left(\operatorname{Im} F^{\prime}\left(x_{0}\right)\right)^{\perp} .
$$

In addition, if $\operatorname{Im} G\left(x_{0}, h\right)=\operatorname{Im} F^{\prime}\left(x_{0}\right) \times Y / \operatorname{Im} F^{\prime}\left(x_{0}\right)$, then $f_{i}(h), i=0,1, \ldots, n$, are not vanishing simultaneously.

\section{THE CASE OF ABNORMAL OPTIMAL CONTROL PROBLEMS}

In this section the results of Section 3 will be used to prove the extended version of the Extremum Principle for Problem $I$.

The classical type Extremum Principle for Problem $I$ was formulated in [13] as an additional case of Papageorgiou's Extremum Principle from [14], by using some version of the Dubovitskii-Milyutin method (Theorem 1 of [9]). However, due to the presence of the fixed initial and terminal data, for some admissible processes $(x, u)$, the Extremum Principle may be 
satisfied for this problem with the trivial multiplier corresponding to the cost functional, $\lambda_{0}=0$. In this case, the adjoint equation and the minimum condition do not depend on the minimized functional $I(x, u)$ and the Extremum Principle describes only the structure of the constraint set, but does not represent much value as a necessary condition of optimality. Processes for which such a degenerate situation occurs are called abnormal.

Definition 4.1: An admissible process $\left(x_{*}, u_{*}\right)$ in Problem $I$ is called abnormal iff there exists a function $p(\cdot) \in W[0, b]$ satisfying the equation

$$
\dot{p}(t)=A_{x}^{*}\left(t, x_{*}(t)\right) p(t)-B_{x}^{*}\left(t, x_{*}(t)\right) u_{*}(t) p(t)
$$

such that

$$
\int_{0}^{b}\left\langle B^{*}\left(t, x_{*}(t)\right) p(t), u_{*}(t)\right\rangle d t=\int_{0}^{b}\left\langle B^{*}\left(t, x_{*}(t)\right) p(t), u\right\rangle d t \quad \text { for all } u \in U(t) .
$$

By applying Theorem 3.1, an extended version of the Extremum Principle to abnormal cases, in the sense of the Definition 4.1, can be proved. In order to apply this theorem to Problem $I$ we need to introduce the following operators:

(i) $\quad F_{1}: W[0, b] \times L^{2}(Y) \rightarrow L^{2}\left(X^{*}\right) \times H$ given by the formula

$$
F_{1}(x, u)(t)=\left(\dot{x}(t)+A(t, x(t))-B(t, x(t)) u(t), x(0)-x_{0}\right) ;
$$

(ii) $\quad F_{2}: W[0, b] \times L^{2}(Y) \rightarrow H$ defined in the form

$$
F_{2}(x, u)=x(b)-x_{1} .
$$

Then we will define the operator $F: W[0, b] \times L^{2}(Y) \rightarrow L^{2}\left(X^{*}\right) \times H \times H$ as

$$
F(x, u)=\left(F_{1}(x, u), F_{2}(x, u)\right)
$$

where $F_{i}(x, u), i=1,2$, are given by formulas (4.1) and (4.2) respectively.

The first and second order Fréchet derivatives of the operator $F$ defined in (4.3) are given by:

$$
\begin{gathered}
F^{\prime}\left(x_{*}, u_{*}\right)(\bar{h}, \bar{v})(t)=\left(\dot{\bar{h}}(t)+A_{x}\left(t, x_{*}(t)\right) \bar{h}(t)-B_{x}\left(t, x_{*}(t)\right) u_{*}(t) \bar{h}(t)\right. \\
\left.-B\left(t, x_{*}(t)\right) \bar{v}, \bar{h}(0), \bar{h}(b)\right), \\
\begin{array}{c}
F^{\prime \prime}\left(x_{*}, u_{*}, h, v\right)(\bar{h}, \bar{v})(t)=\left(A_{x x}\left(t, x_{*}(t)\right) h(t) \bar{h}(t)-B_{x x}\left(t, x_{*}(t)\right) h(t) \bar{h}(t)\right. \\
\left.-B_{x}\left(t, x_{*}(t)\right) v(t) \bar{h}(t)-B_{x}\left(t, x_{*}(t)\right) h(t) \bar{v}(t), 0,0\right) .
\end{array}
\end{gathered}
$$

Then, following Theorem 3.1, let us define the operator 


$$
G\left(x_{*}, u_{*}, h, v\right): W[0, b] \times L^{2}(Y) \rightarrow L^{2}\left(X^{*}\right) \times H \times H \times\left(L^{2}\left(X^{*}\right) \times H \times H\right) / I m F^{\prime}\left(x_{*}, u_{*}\right)
$$

given in the form:

$$
G\left(x_{*}, u_{*}, h, v\right)(\bar{h}, \bar{v})=\left(F^{\prime}\left(x_{*}, u_{*}\right)(\bar{h}, \bar{v}), \pi F^{\prime \prime}\left(x_{*}, u_{*}\right)(h, v)(\bar{h}, \bar{v})\right)
$$

where the Fréchet derivatives $F^{\prime}\left(x_{*}, u_{*}\right)$ and $F^{\prime \prime}\left(x_{*}, u_{*}\right)(h, v)$ are given by (4.4) and (4.5) respectively and $\pi: L^{2}\left(X^{*}\right) \times H \times H \rightarrow L^{2}\left(X^{*}\right) \times H \times H / I m F^{\prime}\left(x_{*}, u_{*}\right)$ is the quotient mapping.

Let us then denote by $P\left(x_{*}, u_{*}\right)$ the set of parameters satisfying the following conditions $(a)-(e)$ :

(a) $\quad \dot{h}+A_{x}\left(t, x_{*}(t)\right) h-B_{x}\left(t, x_{*}(t)\right) u_{*}(t) h-B\left(t, x_{*}(t)\right) v=0$, with $h(0)=0 ;$

(b) $h(b)=0$;

(c) $\quad\left(A_{x x}\left(t, x_{*}(t)\right) h^{2}-B_{x x}\left(t, x_{*}(t)\right) u_{*}(t) h^{2}-B_{x}\left(t, x_{*}(t)\right) v h-B_{x}\left(t, x_{*}(t)\right) h v, 0,0\right) \in$ $\operatorname{Im} F^{\prime}\left(x_{*}, u_{*}\right)$, and $G\left(x_{*}, u_{*}, h, v\right)$ given by (4.6) has a closed image in

$$
\left.L^{2}\left(X^{*}\right) \times H \times H \times\left(L^{2}\left(X^{*}\right) \times H \times H\right) / I m F^{\prime}\left(x_{*}, u_{*}\right)\right)
$$

(d) $\quad \int_{0}^{b}\left(f_{x}\left(x_{*}(t), u_{*}(t), t\right) h(t)+f_{u}\left(x_{*}(t), u_{*}(t), t\right) v(t)\right) d t \leq 0$,

(e) $\quad v$ is such that the second order feasible cone to $V$ at $u_{0}(\cdot)$ in the direction of $v$ is nonempty and convex.

Then, by applying Theorem 3.1, the following extended version of the Extremum Principle can be proved.

\section{Theorem 4.1: (Extremum Principle) Let}

(i) an admissible process $\left(x_{*}, u_{*}\right)$ be optimal in Problem I;

(ii) $\quad\left\|A_{x}\left(t, x_{*}(t)\right)\right\|_{L\left(X, X^{*}\right)} \leq \eta_{1}$;

(iii) $\quad\left\|B_{x}\left(t, x_{*}(t)\right) u_{*}(t)\right\|_{L\left(H, X^{*}\right)} \leq \eta_{2}$;

for some constants $\eta_{1}, \eta_{2}>0$;

(iv) $\quad B_{x}\left(t, x_{*}(t)\right) u_{*}(t) \mid X(\cdot)$ is dissipative;

(v) $\quad t \rightarrow f_{x}\left(t, x_{*}(t), u_{*}(t)\right)$ belongs to $L^{2}(H)$;

(vi) the operator $D: W[0, b] \times L^{2}(Y) \rightarrow L^{2}\left(X^{*}\right) \times H \times H$ in the form

$$
\begin{gathered}
D(\bar{h}, \bar{v})(t)=\left(\dot{\bar{h}}(t)+A_{x}\left(t, x_{*}(t)\right) \bar{h}(t)-B_{x}\left(t, x_{*}(t)\right) u_{*}(t) \bar{h}(t)-B\left(t, x_{*}(t)\right) \bar{v}(t), \bar{h}(0), \bar{h}(b)\right) \\
\text { has a closed image in } L^{2}\left(X^{*}\right) \times H \times H .
\end{gathered}
$$

Then for every $(h, v) \in P\left(x_{*}, u_{*}\right)$ there exist, $\lambda_{0}=\lambda_{0}(h, v) \geq 0$, functions $p(\cdot)=p(h, v)(\cdot)$ and $\psi(\cdot)=\psi(h, v)(\cdot) \in W[0, b]$ not all zero satisfying the "extended adjoint equation"

$$
\dot{p}(t)=\lambda_{0} f_{x}\left(t, x_{*}(t), u_{*}(t)\right)+A_{x}\left(t, x_{*}(t)\right) p(t)-B_{x}\left(t, x_{*}(t)\right) u_{*}(t) p(t)
$$




$$
-A_{x x}^{*}\left(t, x_{*}(t)\right)(h(t)) \psi(t)+B_{x x}^{*}\left(t, x_{*}(t)\right)(h(t)) \psi(t)+B_{x}\left(t, x_{*}(t)\right)(v(t)) \psi(t)
$$

for $t \in[0, b]$ a.e. where the function $\psi(\cdot)$ satisfies

$$
\begin{gathered}
\dot{\psi}(t)=A_{x}\left(t, x_{*}(t)\right) \psi(t)-B_{x}\left(t, x_{*}(t)\right) u_{*}(t) \psi(t) \\
\int_{0}^{b}\left\langle B^{*}\left(t, x_{*}(t)\right)(h(t)) \psi(t), u-u_{*}(t)\right\rangle d t=0 \text { for all } u \in L^{2}(Y)
\end{gathered}
$$

and the "extended minimum condition"

$$
\int_{0}^{b}\left\langle\lambda_{0} f_{u}\left(t, x_{*}(t), u_{*}(t)\right)-B^{*}\left(t, x_{*}(t)\right) p(t)+B_{x}^{*}\left(t, x_{*}(t)\right)(h(t)) \psi(t), u(t)-u_{*}(t)\right\rangle d t \geq 0
$$

for all $u \in V$.

Proof: Our optimal control problem will be formulated in terms of optimization theory as a problem of minimizing the functional $I(x, u)$ under the constraints $(x, u) \in \bigcap_{i=1}^{3} Z_{i}$ where

$$
\begin{gathered}
Z_{1}=\{(x, u): u(\cdot) \in V\} \\
Z_{i+1}=\left\{(x, u): F_{i}(x, u)=0\right\}, i=1,2
\end{gathered}
$$

and $F_{1}$ and $F_{2}$ are given in (4.1) and (4.2) respectively.

We will say that the pair $(x, u) \in W[0, b] \times L^{2}(Y)$ forms an admissible process for Problem $I$ iff $(x, u) \in Z_{i}, i=1,2,3$. In view of the assumptions of the problem, the sets $Z_{2}$ and $Z_{3}$ are equality constraints, i.e. int $Z_{i}=\emptyset, i=2,3$, while $Z_{1}$ is an inequality constraints, i.e. int $Z_{1} \neq \emptyset$. By using Theorem 3.1, the Extremum Principle for Problem $I$ can be proved. In order to apply this theorem, we have to determine the cone of decrease of the functional $I$ at the point $\left(x_{*}, u_{*}\right)$ denoted by $D C\left(I,\left(x_{*}, u_{*}\right)\right.$, the feasible cone to the set $Z_{1}$ at the point $\left(x_{*}, u_{*}\right)$ denoted by $F C\left(Z_{1},\left(x_{*}, u_{*}\right)\right.$ and the cones dual to them.

Following well known arguments as they can be found in [14], we get that

$$
\begin{gathered}
D C\left(I,\left(x_{*}, u_{*}\right)\right)=\left\{(\bar{h}, \bar{v}) \in W[0, b] \times L^{2}(Y): \int_{0}^{b}\left(f_{x}\left(t, x_{*}(t), u_{*}(t)\right) \bar{h}(t)\right.\right. \\
\left.\left.+f_{u}\left(t, x_{*}(t), u_{*}(t)\right) \bar{v}(t)\right) d t<0\right\} \\
\left(D C\left(I,\left(x_{*}, u_{*}\right)\right)^{*}=\left\{f_{0}(\bar{h}, \bar{v})=-\lambda_{0} \int_{0}^{b}\left(f_{x}\left(t, x_{*}(t), u_{*}(t)\right) \bar{h}(t)+f_{u}\left(t, x_{*}(t), u_{*}(t)\right) \bar{v}(t) d t\right.\right.\right.
\end{gathered}
$$




$$
\text { where } \left.\lambda_{0} \geq 0\right\}
$$

Then let us analyze the inequality constraint $Z_{1}$ given by (4.11). Theorem 10.5 of [4] implies that

$$
F C\left(Z_{1},\left(x_{*}, u_{*}\right)\right)^{*}=\left\{f_{1}=\left(0, f_{1}^{\prime}\right): f_{1}(\bar{h}, \bar{v})=f_{1}^{\prime}(\bar{v})\right.
$$

where $f_{1}^{\prime}(\bar{v})$ is a functional supporting the set $V$ at the point $\left.u_{*}\right\}$.

Finally, consider the operator $F$ defined by (4.3). Since $F_{1}$ and $F_{2}$ are continuously Fréchet differentiable at $\left(x_{*}, u_{*}\right)$, so is $F$. Its derivative at this point has the form of the operator $D$ from assumption (vi). In view of $(i i)$, the derivative of $F$ has then a closed image in $H \times H \times L^{2}\left(X^{*}\right)$. Thus all the assumptions of Theorem 3.1 are satisfied. Then, in view of this theorem, for every $(h, v) \in P\left(x_{*}, u_{*}\right)$ there exist functionals, not all zero, $f_{0}(h, v) \in$ $\left(D C\left(I,\left(x_{*}, u_{*}\right)\right)\right)^{*}, f_{1}(h, v) \in\left(F C\left(Z_{1},\left(x_{*}, u_{*}\right)\right)\right)^{*}, q_{j}^{*}(h, v), s_{j}^{*}(h, v) \in Y_{j}^{*}, j=1,2$, such that

$$
\begin{gathered}
f_{0}(h)+f_{1}(h)+F_{1}^{\prime *}\left(x_{0}\right) q_{1}^{*}(h)+F_{2}^{\prime *}\left(x_{0}\right) q_{2}^{*}(h)+\left(F_{1}^{\prime \prime}\left(x_{0}\right)(h)\right)^{*} s_{1}^{*}(h) \\
+\left(F_{2}^{\prime \prime}\left(x_{0}\right)(h)\right)^{*} s_{2}^{*}(h)=0
\end{gathered}
$$

and

$$
\left(s_{1}^{*}(h, v), s_{2}^{*}(h, v)\right) \in\left(\operatorname{Im} F^{\prime}\left(x_{*}, u_{*}\right)\right)^{\perp} .
$$

By using (4.4), (4.5), (4.13) and (4.14) and forms of the linear functionals on $H$ and $L^{2}\left(H^{*}\right)$, the Euler-Lagrange equations will have the form

$$
\begin{gathered}
-\lambda_{0} \int_{0}^{b}\left(f_{x}\left(t, x_{*}(t), u_{*}(t)\right) \bar{h}(t)+f_{u}\left(t, x_{*}(t), u_{*}(t)\right) \bar{v}(t)\right) d t+f_{1}^{\prime}(\bar{v}) \\
+\int_{0}^{b}\left\langle\dot{\bar{h}}(t)+A_{x}\left(t, x_{*}(t)\right) \bar{h}(t)-B_{x}\left(t, x_{*}(t)\right) u_{*}(t) \bar{h}(t)-B\left(t, x_{*}(t)\right) \bar{v}(t), p(t)\right\rangle d t+(a, \bar{h}(t)(0)) \\
+(b, \bar{h}(b))+\int_{0}^{b}\left\langle A_{x x}\left(t, x_{*}(t)\right) h(t) \bar{h}(t)-B_{x x}\left(t, x_{*}(t)\right) h(t) \bar{h}(t)-B_{x}\left(t, x_{*}(t)\right) v(t) \bar{h}(t)\right. \\
\left.-B_{x}\left(t, x_{*}(t)\right) h(t) \bar{v}(t), \psi(t)\right\rangle d t=0
\end{gathered}
$$

for every $(h, v) \in P\left(x_{*}, u_{*}\right)$ and $(\bar{h}, \bar{v}) \in W[0, b] \times L^{2}(Y)$, where $p(\cdot), \psi(\cdot) \in L^{2}\left(X^{*}\right), a, b \in H$.

Similarly, applying (4.4) in (4.16) and using the definition of the annihilator of the subspace, we obtain that $\psi(\cdot)$ satisfies

$$
\int_{0}^{b}\left\langle\psi(t), \dot{h}(t)+A_{x}\left(t, x_{*}(t)\right) h(t)-B_{x}\left(t, x_{*}(t)\right) u_{*}(t) h(t)-B\left(t, x_{*}(t)\right) v(t)\right\rangle d t
$$




$$
+(c, h(0))+(d, h(b))=0 .
$$

with $c$ and $d$ some elements of the space $H$.

First let us consider (4.17) for $\bar{v}=0$. Changing $p(t)$ on a set of measure zero, if necessary, we obtain the existence of $p(t) \in W[0, T]$. Applying in (4.17) Lemma 5.1 of [17] about integration by parts and using the definition of adjoint operators for $A_{x}, A_{x x}$ and $B_{x}$, $B_{x x}$ we get

$$
\begin{gathered}
\int_{0}^{b}\left\langle-\dot{p}(t)-\lambda_{0} f_{x}\left(t, x_{*}(t), u_{*}(t)\right)+A_{x}\left(t, x_{*}(t)\right) p(t)-B_{x}\left(t, x_{*}(t)\right) u_{*}(t) p(t)\right. \\
\left.+A_{x x}^{*}\left(t, x_{*}(t)\right)(h(t)) \psi(t)-B_{x x}^{*}\left(t, x_{*}(t)\right)(h(t)) \psi(t)-B_{x}\left(t, x_{*}(t)\right)(v(t)) \psi(t), \bar{h}(t)\right\rangle d t \\
+(a-p(0), \bar{h}(0))+(b+p(b), \bar{h}(b))=0 .
\end{gathered}
$$

Equation (4.19) considered for $\bar{h}(0)=0$ and $\bar{h}(b)=0$ implies the "extended" adjoint equation (4.8). Taking in (4.17) $\bar{h}=0$ and using the definition of the adjoint operators $B$ and $B_{x}$ we obtain

$$
f_{1}^{\prime}(\bar{v})=\int_{0}^{b}\left\langle\lambda_{0} f_{u}\left(t, x_{*}(t), u_{*}(t)\right)-B^{*}\left(t, x_{*}(t)\right) p(t)+B_{x}^{*}\left(t, x_{*}(t)\right)(h(t)) \psi(t), \bar{v}(t)\right\rangle d t .
$$

Then from the definition of a functional supporting the set $U$ at $u_{*}(\cdot)$, from (4.20) the "extended minimum condition" (4.10) of this theorem follows.

This theorem has been proved under the assumption that $D C\left(I,\left(x_{*}, u_{*}\right)\right) \neq \emptyset$. If this assumption is not true, then from the form of $D C\left(I,\left(x_{*}, u_{*}\right)\right)$ it follows that for every $\left(\bar{h}(t), \bar{v}(t) \in W[0, b] \times L^{2}(Y)\right.$ we have

$$
\int_{0}^{b}\left(f_{x}\left(t, x_{*}(t), u_{*}(t)\right) \bar{h}(t)+f_{u}\left(t, x_{*}(t), u_{*}(t)\right) \bar{v}(t)\right) d t=0 .
$$

Putting in (4.21) $\lambda_{0}=1$ and taking $\psi \equiv 0, p \equiv 0, a=0, b=0, f_{1}^{\prime}=0$, we obtain the Euler-Lagrange equation (4.17) and then proceeding in the same way as in the first part of the proof we obtain the proposition.

Finally let's consider (4.18) for $\bar{v}=0$. Changing $\psi(t)$ on a set of measure zero, if necessary, we obtain the existence of $\psi(t) \in W[0, T]$. Applying in (4.18) Lemma 5.1 of [17] about the integration by parts and using the definition of adjoint operators for $A_{x}, B_{x}$ and $B$ we get

$$
\int_{0}^{b}\left\langle-\dot{\psi}(t)+A_{x}\left(t, x_{*}(t)\right) \psi(t)-B_{x}\left(t, x_{*}(t)\right) u_{*}(t) \psi(t), \bar{h}(t)\right\rangle d t
$$




$$
+(c-\psi(0), \bar{h}(0))+(d+\psi(b), \bar{h}(b))=0 .
$$

Taking (4.19) for $\bar{h}(0)=0$ and $\bar{h}(b)=0$ we obtain equation (4.9a). Then substituting in (4.18) $\bar{h}=0$ and using the adjoint operator $B$ we obtain condition (4.9b).

Q.E.D.

Remark 4.1: If we assume in addition that $U(\cdot)$ is $L_{+}^{2}$ bounded in the sense that the mapping

$$
t \rightarrow|u(t)|=\sup \{\|u\|: u \in U(t)\} \text { belongs to } L_{+}^{2},
$$

then, proceeding analogously as in Theorem 3.1 of [14] we can derive that the minimum condition (4.10) can be expressed in the simpler form

$$
\left\langle\lambda_{0} f_{u}\left(t, x_{*}(t), u_{*}(t)\right)-B^{*}\left(t, x_{*}(t)\right) p(t), u-u_{*}(t)\right\rangle \geq 0
$$

for all $u \in U(t)$ and a.e. $t \in[0, b]$.

Remark 4.2: If $U=L^{2}(Y)$, then condition (2.5) has the form $u(\cdot) \in L^{2}(Y)$, i.e. there are no constraints on the control functions. Then the minimum condition (4.23) of Remark 4.1 takes the form

$$
\lambda_{0} f_{u}\left(t, x_{*}(t), u_{*}(t)\right)=B^{*}\left(t, x_{*}(t)\right) p(t) \text { a.e. }
$$

Remark 4.3: $\quad$ If in assumption (vi) $\operatorname{ImD}=L^{2}\left(X^{*}\right) \times H \times H$ (the regular case), then from condition (4.9) we have that $\psi(\cdot) \equiv 0$ and in Theorem 4.1 the following changes occur:

a) conditions $(4.9 a),(4.9 b)$ disappear;

b) the "extended adjoint equation" (4.8) takes the form of the classical "adjoint equation"

$$
\dot{p}(t)=\lambda_{0} f_{x}\left(t, x_{*}(t), u_{*}(t)\right)+A_{x}\left(t, x_{*}(t)\right) p(t)-B_{x}\left(t, x_{*}(t)\right) u_{*}(t) p(t), \text { a.e. }
$$

c) the "extended minimum condition" takes the form of the classical "minimum condition"

$$
\int_{0}^{b}\left\langle\lambda_{0} f_{u}\left(t, x_{*}(t), u_{*}(t)\right)-B^{*}\left(t, x_{*}(t)\right) p(t), u(t)-u_{*}(t)\right\rangle d t \geq 0 \text { for } u(\cdot) \in V .
$$




\section{AN OPTIMAL CONTROL PROBLEM WITH NONOPERATOR EQUALITY CONSTRAINTS}

Let us now consider Problem $I$ without the terminal data (2.4), but also without assumption $(A 11)$ that the set $V$ possesses a nonempty interior in $L^{2}(Y)$. Let us call such a problem, Problem II. Under these changes the geometrical model of the problem, by using the Dubovitskii-Milyutin approach, will change because we will have two equality constraints $Z_{1}$ and $Z_{2}$ given by (4.11) and (4.12) respectively. The situation here will be different than in Problem $I$, because the constraints $Z_{1}$ is firstly, an equality constraint and secondly, it is in the nonoperator form. In order to prove the Extremum Principle for Problem $I I$ we have to apply some generalization of the Dubovitskii-Milyutin method to the case of $n$ equality constraints in the arbitrary, even nonoperator form from [15].

Theorem 5.1: (Extremum Principle) Let the admissible process $\left(x_{*}, u_{*}\right)$ be optimal for Problem II and suppose assumptions (ii)-(v) of Theorem 4.1 are satisfied. Then there exist $\lambda_{0} \geq 0$ and $p(\cdot) \in W[0, b]$ not all zero satisfying the "adjoint equation" (4.24) with terminal condition $p(b)=0$, such that the "minimum condition" (4.25) holds.

Proof: In the proof we will use Theorem 4.1 of [15]. Assume first that the cone $D C\left(I,\left(x_{*}, u_{*}\right)\right) \neq \emptyset$. We will be able to use the form $(4.13)$ of the cone $\left(D C\left(I,\left(x_{*}, u_{*}\right)\right)\right)^{*}$. By using Theorem 4.2 of [3] it is easy to see that $F_{1}^{\prime}\left(x_{*}, u_{*}\right)$ given as

$$
F_{1}^{\prime}\left(x_{*}, u_{*}\right)(\bar{h}, \bar{v})(t)=\left(\dot{\bar{h}}(t)+A_{x}\left(t, x_{*}(t)\right) \bar{h}(t)-B_{x}\left(t, x_{*}(t)\right) u_{*}(t) \bar{h}(t)-B\left(t, x_{*}(t)\right) \bar{v}(t) \bar{h}(0)\right)
$$

maps $W[0, b] \times L^{2}(Y)$ onto the space $L^{2}\left(X^{*}\right) \times H$. Then applying the classical Lusternik Theorem to the set $Z_{2}$ given by (4.2), we get that

$$
\begin{gathered}
T C\left(Z_{2},\left(x_{*}, u_{*}\right)\right)=\left\{(\bar{h}, \bar{v}): \dot{\bar{h}}(t)+A_{x}\left(t, x_{*}(t)\right) \bar{h}(t)-B_{x}\left(t, x_{*}(t)\right) u_{*}(t) \bar{v}(t)-B\left(t, x_{*}(t)\right) \bar{v}(t)=0,\right. \\
\bar{h}(0)=0\} .
\end{gathered}
$$

Notice that, we don't need to determine the dual cone to $T C\left(Z_{2},\left(x_{*}, u_{*}\right)\right)$ in the proof.

From the form (4.11) of the equality constraint $Z_{1}$, we obtain that

$$
T C\left(Z_{1},\left(x_{*}, u_{*}\right)\right)=W[0, b] \times \bar{C}_{1}
$$

where $\bar{C}_{1} \subseteq L^{2}(Y)$ denotes the tangent cone to the set $V$ at the point $u_{*}$. On the basis of Theorem 10.5 of [4], the cone $\left(T C\left(Z_{1},\left(x_{*}, u_{*}\right)\right)\right)^{*}$ is given by (4.14). 
In order to apply Theorem 4.1 of [15] we have to show that, similarly as in [16], the cones $\left(T C\left(Z_{1},\left(x_{*}, u_{*}\right)\right)\right)^{*}$ and $\left(T C\left(Z_{2},\left(x_{*}, u_{*}\right)\right)\right)^{*}$ are of "the same sense", according to Definition 2.1 of [15]. In order to show this we have to analyze the form of the cones $T C\left(Z_{1},\left(x_{*}, u_{*}\right)\right)$ given by $(4.14)$ and $T C\left(Z_{2},\left(x_{*}, u_{*}\right)\right)$ given by (5.2). By applying Theorem 4.2 of [3] about the existence and uniqueness of solution to the following equation from (4.14)

$$
\dot{\bar{h}}^{(t)}+A_{x}\left(t, x_{*}(t)\right) \bar{h}^{(t)}-B_{x}\left(t, x_{*}(t)\right) u_{*}(t) \bar{v}^{(t)}-B\left(t, x_{*}(t)\right) \bar{v}^{(t)}=0, \quad \bar{h}(0)=0,
$$

we have that for every $\bar{v} \in L^{2}(Y)$ there exists a unique $\bar{h} \in W[0, b]$ satisfying (5.4). From above and from the assumptions $(A 2)-(A 8)$ about operators $A_{x}\left(t, x_{*}(t)\right), B_{x}\left(t, x_{*}(t)\right)$ and $B\left(t, x_{*}(t)\right)$ we obtain that $T C\left(Z_{2},\left(x_{*}, u_{*}\right)\right)$ can be expressed in the form

$$
T C\left(Z_{2},\left(x_{*}, u_{*}\right)\right)=\{(\bar{h}, \bar{v}): \bar{h}=M \bar{v}\},
$$

where $M: L^{2}(Y) \rightarrow W[0, b]$ is a linear and continuous operator such that $\bar{h}=M \bar{v}$ iff $(\bar{h}, \bar{v})$ satisfies equations in (5.2).

Applying then Theorem 3.1 of [15] to the cones given by (5.3) and (5.5) we obtain that the cones $\left(T C\left(Z_{1},\left(x_{*}, u_{*}\right)\right)\right)^{*}$ and $\left(T C\left(Z_{4},\left(x_{*}, u_{*}\right)\right)\right)^{*}$ are of "the same sense" i.e. assumption (iv) of Theorem 4.1 of [15] is satisfied.

Assumptions $(i)$ and $(i i)$ of this theorem are also satisfied, since the cones $D C\left(I,\left(x_{*}, u_{*}\right)\right), T C\left(Z_{1},\left(x_{*}, u_{*}\right)\right)$ and $T C\left(Z_{2},\left(x_{*}, u_{*}\right)\right)$ are convex. Then we only have to show that assumption (iii) of this theorem is satisfied i.e.

$$
T C\left(Z_{1},\left(x_{*}, u_{*}\right)\right) \cap T C\left(Z_{2},\left(x_{*}, u_{*}\right)\right) \subseteq T C\left(Z_{1} \cap Z_{2},\left(x_{*}, u_{*}\right)\right) .
$$

The proof of this fact will be similar to the one given in [16]. Consider $Z_{2}$ given by (4.12), where the operator $F_{1}$ is given by (4.1). It is easy to check that in some neighborhood $\mathcal{U}$ of the point $\left(x_{*}, u_{*}\right)$ the operator $F_{1}$ satisfies all the assumptions of the implicit function theorem. Then, in this neighborhood $\mathcal{U}$, the set $Z_{2}$ can be expressed in the form

$$
Z_{2}=\{(x, u): x=S(u)\}
$$

where $S: L^{2}(Y) \rightarrow W[0, b]$ is an operator of the class $C^{1}$ satisfying the condition $F_{1}(S(u), u)=0$ for every $u$ such that $(S(u), u) \in \mathcal{U}$. Differentiating the above we obtain that the cone $T C\left(Z_{2},\left(x_{*}, u_{*}\right)\right)$ can be represented in the form

$$
T C\left(Z_{2},\left(x_{*}, u_{*}\right)\right)=\left\{(\bar{h}, \bar{v}) \in W[0, b] \times L^{2}(Y): \bar{h}=S_{u}\left(u_{*}\right) \bar{v}\right\}
$$


Let us take an arbitrary $(\bar{h}, \bar{v}) \in T C\left(Z_{1},\left(x_{*}, u_{*}\right)\right) \cap T C\left(Z_{2},\left(x_{*}, u_{*}\right)\right)$. Then from the definition of the tangent direction, there exists an operator $r_{u}^{1}(t)$ such that $\left\|r_{u}^{1}(t)\right\| / t \rightarrow 0$ as $t \rightarrow 0^{+}$and

$$
\left(x_{*}, u_{*}\right)+t(\bar{x}, \bar{u})+\left(r_{x}^{1}(t), r_{u}^{1}(t)\right) \in Z_{1}
$$

for sufficiently small $t$ and any $\left(r_{x}^{1}(t)\right)$. Then from (5.7), for sufficiently small $t$, we have

$$
\left(S\left(u_{*}+t \bar{u}+r_{u}^{1}(t)\right), u_{*}+t \bar{u}+r_{u}^{1}(t)\right) \in Z_{1} .
$$

Since $S$ is Fréchet differentiable, there exists an operator $r_{x}^{2}(t)$ such that $\left\|r_{x}^{2}(t)\right\| / t \rightarrow 0$ as $t \rightarrow 0^{+}$and

$$
S\left(u_{*}+t \bar{u}+r_{u}^{1}(t)\right)=S\left(u_{*}\right)+t S_{u}\left(u_{*}\right) \bar{u}+r_{x}^{2}(t)
$$

Then using (5.8) we get

$$
\left(x_{*}, u_{*}\right)+t(\bar{x}, \bar{u})+\left(r_{x}^{1}(t), r_{u}^{1}(t)\right) \in Z_{2} .
$$

Now it is enough to take in (5.9) $r_{x}^{1}(t)=r_{x}^{2}(t)$ and then (5.10)-(5.12) imply that (5.6) holds. So all the assumptions of Theorem 4.1 of [15] are satisfied. By making use of this theorem we obtain that there exist functionals: $f_{0} \in\left(D\left(I,\left(x_{*}, u_{*}\right)\right)\right)^{*}, f_{i} \in T C\left(Z_{i},\left(x_{*}, u_{*}\right)\right)$, $i=1,2$, not all zero, such that

$$
f_{0}(\bar{h}, \bar{v})+f_{1}(\bar{h}, \bar{v})+f_{2}(\bar{h}, \bar{v})=0 .
$$

The proposition now follows as in [14], using a classical Dubovitskii-Milyutin framework (e.g. [4]). So does the case when $D C\left(I,\left(x_{*}, u_{*}\right)\right)=\emptyset$, which ends the proof.

Remark 5.1: It is worth to notice that Remark 4.1 remains true for Problem $I I$, i.e. in the cases described in Remark 4.1 the minimum condition can be simplified to the form (4.23).

Remark 5.2: As an example of the Theorem 5.1 we can consider optimal control problems of systems governed by partial differential equations discussed in [10]. The Extremum Principle proved in [10] now becomes a simple corollary of Theorem 5.1.

\section{REFERENCES}

[1] Avakov, E.R, Necessary extremum conditions for smooth abnormal problems with equality and inequality-type constraints, Matematischeskie Zametki Vol. 45, No. 6, pp. 3-11, (June 1989). 
[2] Avakov, E.R., Necessary conditions for the minimum for the nonregular problems in Banach spaces. Maximum principle for abnormal problems of optimal control. Trudy Matematiceskovo Instiuta, AN-SSSR Vol. 185, pp. 3-29, (1988), in Russian.

[3] Barbu, V., Nonlinear Semigroups and Differential Equations in Banach Spaces, Noordhoff, The Netherlands 1976.

[4] Girsanov, I.V., Lectures on Mathematical Theory of Extremum Problems, SpringerVerlag, New York, New York 1972.

[5] Kotarski, W., and A. Kowalewski, On optimal control problem with initial state not a prior given, Problems of Control and Information Theory 12, (1983), pp. 349-359.

[6] Kotarski, W., Optimal control of a system governed by a parabolic equation with an infinite number of variables and time delay, J. of Optimization Theory and Applications 63, (1989), pp. 57-67.

[7] Lions, J.L., Optimal Control of Systems Governed by Partial Differential Equations, Springer-Verlag, New York, New York 1971.

[8] Lions, J.L., Optimal control of non well-posed distributed systems, Mathematical Control Theory, Banach Center Publications 14, (1985), pp. 299-311 (Polish Scientific Publishers, Warsaw).

[9] Ledzewicz-Kowalewska, U., On some specification of the Dubovitskii-Milyutin method, J. of Nonlinear Anal. 12, (1986), pp. 1367-1371.

[10] Ledzewicz-Kowalewska, U., The necessary and sufficient condition of the problems of optimal control of parabolic systems with equality constraints, Bulletin de La Societe and des sciences et des Lettres de Lodz XXXVI, (1986), pp. 1-12.

[11] Ledzewicz, U., and Schaettler, H., Second order conditions for Extremum problems with nonregular equality constraints, to appear.

[12] Ledzewicz, U., Extension of the local minimum principle to abnormal optimal control problems, J. of Optimization Theory and Applications.

[13] Ledzewicz-Kowalewska, U., The Extremum Principle for some types of distributed parameter systems, Applicable Analysis, 48, (1993), pp.1-21..

[14] Papageorgiou, N.S., Optimality conditions for systems with insufficient data, Bull. Austral. Math. Soc. Vol. 11, (1990), pp. 45-55.

[15] Walczak, S., On some properties of cones in normed spaces and their applications to investigating Extremal problems, J. Optimization Theory and Applications 42, (1984), pp. 561-582.

[16] Walczak, S., On some control problem, Acta Universitatis Lodziensis, Folia Mathematica 1, 1984.

[17] Tanabe, H., Equations of Evolution, Pitman, London 1979. 


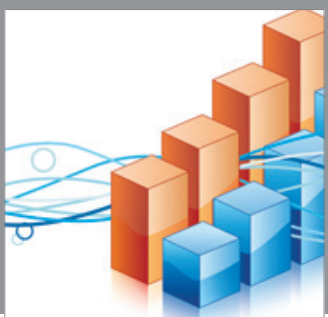

Advances in

Operations Research

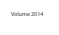

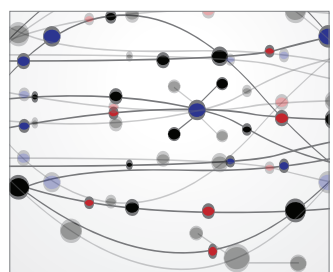

\section{The Scientific} World Journal
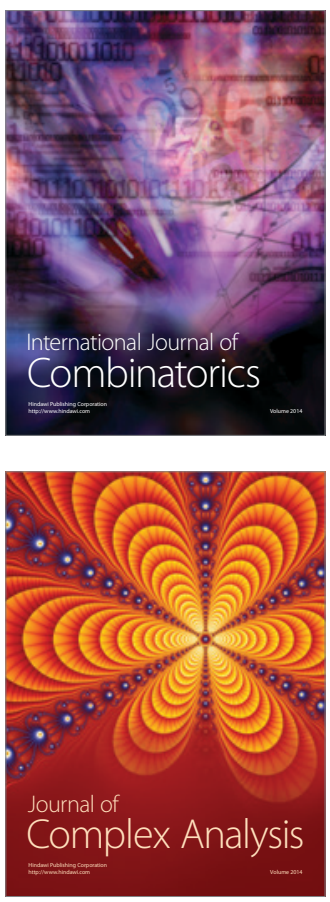

International Journal of

Mathematics and

Mathematical

Sciences
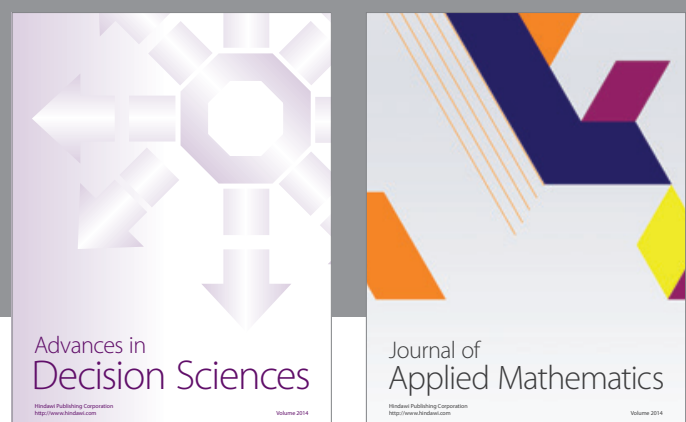

Journal of

Applied Mathematics
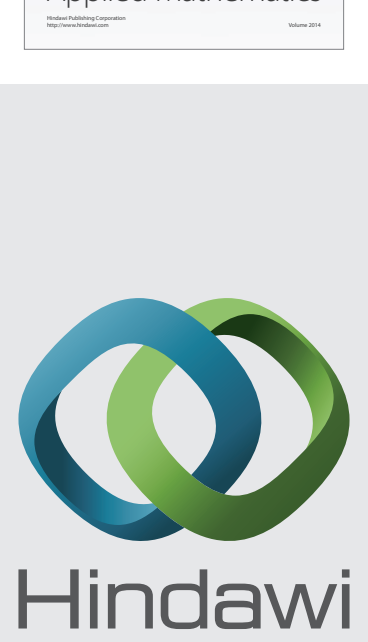

Submit your manuscripts at http://www.hindawi.com
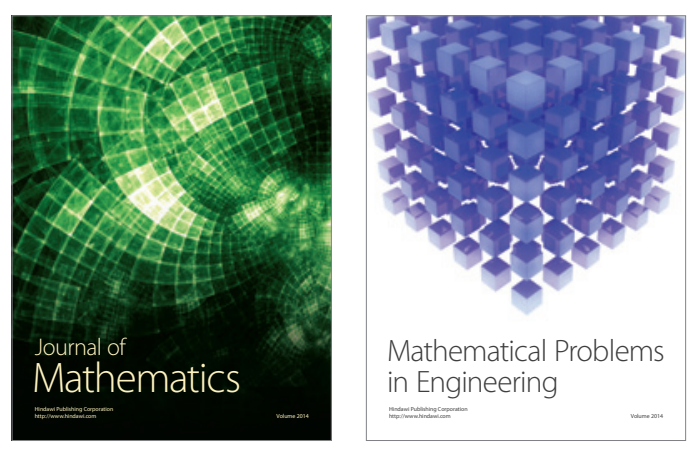

Mathematical Problems in Engineering
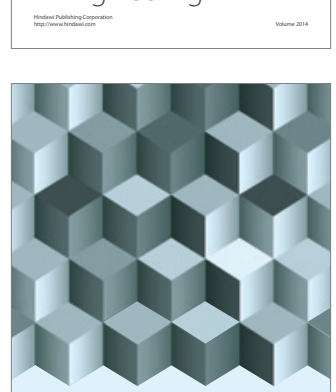

Journal of

Function Spaces
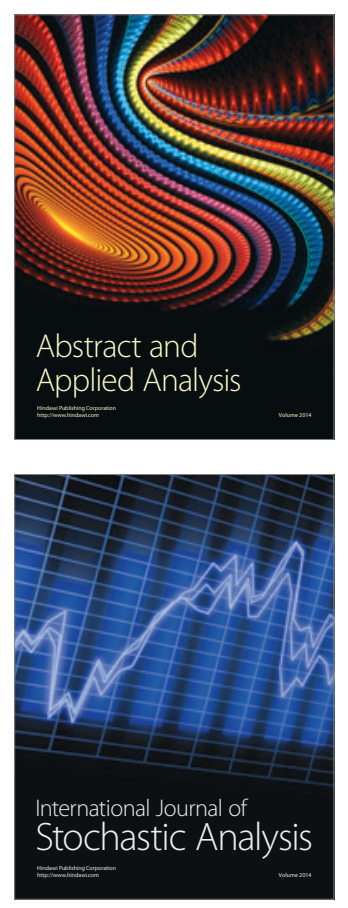

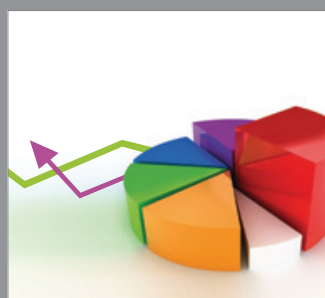

ournal of

Probability and Statistics

Promensencen
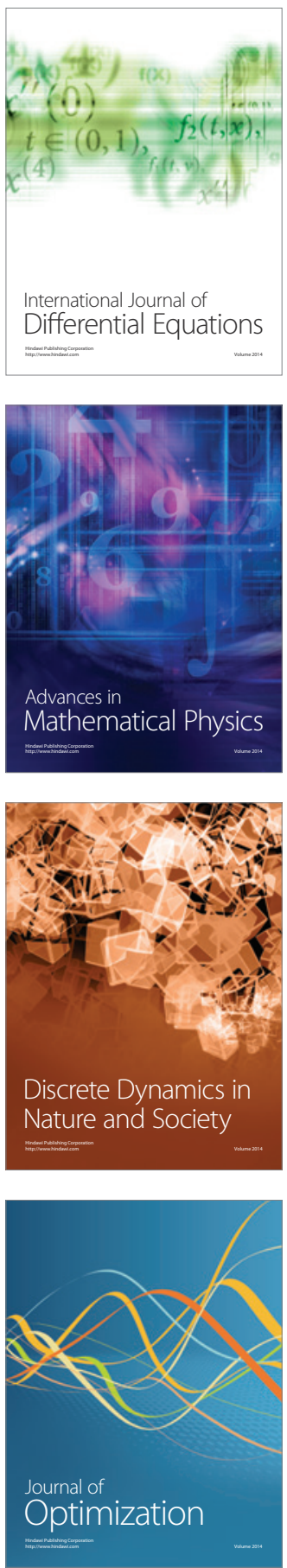